\title{
On new inequalities of Hermite-Hadamard-Fejer type for quasi-geometrically convex functions via fractional integrals
}

\author{
Mehmet Kunt* and İmdat İşcan \\ (Communicated by Nihal YILMAZ ÖZGÜR)
}

\begin{abstract}
In this paper, new Hermite-Hadamard-Fejer type integral inequalities for quasi-geometrically convex functions in fractional integral forms are obtained.
\end{abstract}

Keywords: Hermite-Hadamard inequality; Hermite-Hadamard-Fejer inequality; Hadamard fractional integral; quasigeometrically convex function.

AMS Subject Classification (2010): Primary: 26A51; Secondary: 26A33; 26D10.

${ }^{*}$ Corresponding author

\section{Introduction}

Let $f: I \subseteq \mathbb{R} \rightarrow \mathbb{R}$ be a convex function defined on the interval $I$ of real numbers and $a, b \in I$ with $a<b$. The inequality

$$
f\left(\frac{a+b}{2}\right) \leq \frac{1}{b-a} \int_{a}^{b} f(x) d x \leq \frac{f(a)+f(b)}{2}
$$

is well known in the literature as Hermite-Hadamard's inequality [2].

The most well-known inequalities related to the integral mean of a convex function $f$ are the Hermite Hadamard inequalities or their weighted versions, the so-called Hermite-Hadamard-Fejér inequalities.

In [1], Fejér established the following Fejér inequality which is the weighted generalization of Hermite-Hadamard inequality (1.1):

Theorem 1. Let $f:[a, b] \rightarrow \mathbb{R}$ be a convex function. Then the inequality

$$
f\left(\frac{a+b}{2}\right) \int_{a}^{b} g(x) d x \leq \int_{a}^{b} f(x) g(x) d x \leq \frac{f(a)+f(b)}{2} \int_{a}^{b} g(x) d x
$$

holds, where $g:[a, b] \rightarrow \mathbb{R}$ is nonnegative, integrable and symmetric to $(a+b) / 2$.

For some results which generalize, improve and extend the inequalities (1.1) and (1.2) see [3, 12-14].

Definition 1. [10, 11]. A function $f: I \subset(0, \infty) \rightarrow \mathbb{R}$ is said to be GA-convex (geometric-arithmetically convex) if

$$
f\left(x^{t} y^{1-t}\right) \leq t f(x)+(1-t) f(y)
$$

for all $x, y \in I$ and $t \in[0,1]$.

Received : 22-July-2015, Accepted : 29-April-2016 
Definition 2. [5]. A function $f: I \subset(0, \infty) \rightarrow \mathbb{R}$ is said to be quasi-geometrically convex on $I$ if

$$
f\left(x^{t} y^{1-t}\right) \leq \sup \{f(x), f(y)\}
$$

for all $x, y \in I$ and $t \in[0,1]$.

In [9], Latif et al. established following Hermite-Hadamard-Fejer type inequality for GA-convex functions as follows:

Theorem 2. Let $f: I \subset(0, \infty) \rightarrow \mathbb{R}$ be a GA-convex function and $a, b \in I$ with $a<b$. Let $g:[a, b] \rightarrow[0, \infty)$ be continuous positive mapping and geometrically symmetric to $\sqrt{a b}$. Then

$$
f(\sqrt{a b}) \int_{a}^{b} \frac{g(x)}{x} d x \leq \int_{a}^{b} \frac{f(x) g(x)}{x} d x \leq \frac{f(a)+f(b)}{2} \int_{a}^{b} \frac{g(x)}{x} d x .
$$

In [8], Kunt et al. established following Hermite-Hadamard and Hermite-Hadamard-Fejer type inequality for GA-convex function in fractional integral forms as follows:

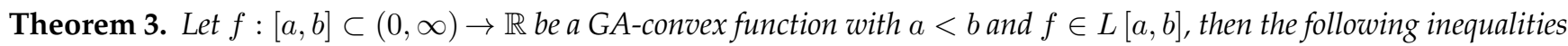
for fractional integrals holds:

$$
f(\sqrt{a b}) \leq \frac{\Gamma(\alpha+1)}{2^{1-\alpha}\left(\ln \frac{b}{a}\right)^{\alpha}}\left[J_{\sqrt{a b}-}^{\alpha} f(a)+J_{\sqrt{a b}+}^{\alpha} f(b)\right] \leq \frac{f(a)+f(b)}{2} .
$$

with $\alpha>0$.

Theorem 4. Let $f:[a, b] \subset(0, \infty) \rightarrow \mathbb{R}$ be a GA-convex function with $a<b$ and $f \in L[a, b]$. If $g:[a, b] \rightarrow \mathbb{R}$ is nonnegative, integrable and geometrically symmetric with respect to $\sqrt{a b}$, then the following inequalities for fractional integrals holds:

$$
\begin{aligned}
f(\sqrt{a b})\left[J_{\sqrt{a b}-}^{\alpha} g(a)+J_{\sqrt{a b}+}^{\alpha} g(b)\right] & \leq\left[J_{\sqrt{a b}-}^{\alpha}(f g)(a)+J_{\sqrt{a b}+}^{\alpha}(f g)(b)\right] \\
& \leq \frac{f(a)+f(b)}{2}\left[J_{\sqrt{a b}-}^{\alpha} g(a)+J_{\sqrt{a b}+}^{\alpha} g(b)\right]
\end{aligned}
$$

with $\alpha>0$.

Lemma 1. [8]. Let $f:[a, b] \subset(0, \infty) \rightarrow \mathbb{R}$ be a differentiable mapping on $(a, b)$ with $a<b$ and $f^{\prime} \in L[a, b]$. If $g:[a, b] \rightarrow \mathbb{R}$ is integrable and geometrically symmetric with respect to $\sqrt{a b}$ then the following equality for fractional integrals holds:

$$
\left[\begin{array}{c}
f(\sqrt{a b})\left[J_{\sqrt{a b}-}^{\alpha} g(a)+J_{\sqrt{a b}+}^{\alpha} g(b)\right] \\
-\left[J_{\sqrt{a b}-}^{\alpha}(f g)(a)+J_{\sqrt{a b}+}^{\alpha}(f g)(b)\right.
\end{array}\right]=\frac{1}{\Gamma(\alpha)}\left[\begin{array}{c}
\int_{a}^{\sqrt{a b}}\left(\int_{a}^{t}\left(\ln \frac{s}{a}\right)^{\alpha-1} g(s) \frac{d s}{s}\right) f^{\prime}(t) d t \\
+\int_{\sqrt{a b}}^{b}\left(\int_{t}^{b}\left(\ln \frac{b}{s}\right)^{\alpha-1} g(s) \frac{d s}{s}\right) f^{\prime}(t) d t
\end{array}\right]
$$

with $\alpha>0$.

The following definitions and mathematical preliminaries of fractional calculus theory are used further in this paper.

Definition 3. [7]. Let $f \in L[a, b]$. The Hadamard integrals $J_{a+}^{\alpha} f$ and $J_{b-}^{\alpha} f$ of order $\alpha>0$ with $a \geq 0$ are defined by

$$
J_{a+}^{\alpha} f(x)=\frac{1}{\Gamma(\alpha)} \int_{a}^{x}\left(\ln \frac{x}{t}\right)^{\alpha-1} f(t) \frac{d t}{t}, x>a \text { and } J_{b-}^{\alpha} f(x)=\frac{1}{\Gamma(\alpha)} \int_{x}^{b}\left(\ln \frac{t}{x}\right)^{\alpha-1} f(t) \frac{d t}{t}, x<b
$$

respectively, where $\Gamma(\alpha)$ is the Gamma function defined by $\Gamma(\alpha)=\int_{0}^{\infty} e^{-t} t^{\alpha-1} d t$.

Because of the wide application of Hermite-Hadamard type inequalities and fractional integrals, many researchers extend their studies to Hermite-Hadamard type inequalities involving fractional integrals not limited to integer integrals. Recently, more and more Hermite-Hadamard inequalities involving fractional integrals have been obtained for different classes of functions; see [4-6, 15, 16].

In this paper, we obtain some Hermite-Hadamard-Fejer type integral inequalities for quasi-geometrically convex functions in fractional integral forms. 


\section{Main results}

Throughout this section, we write $\|g\|_{\infty}=\sup _{t \in[a, b]}|g(t)|$, for the continuous function $g:[a, b] \rightarrow \mathbb{R}$.

Theorem 5. Let $f: I \subset(0, \infty) \rightarrow \mathbb{R}$ be a differentiable function on $I^{\circ}$, the interior of $I$, such that $f^{\prime} \in L[a, b]$, where $a, b \in I$ and $a<b$. If $\left|f^{\prime}\right|$ is quasi-geometrically convex on $[a, b], g:[a, b] \rightarrow \mathbb{R}$ is continuous and geometrically symmetric with respect to $\sqrt{a b}$, then the following inequality for fractional integrals holds:

$$
\left.\left[\mid \begin{array}{l}
f(\sqrt{a b})\left[J_{\sqrt{a b}-}^{\alpha} g(a)+J_{\sqrt{a b}+}^{\alpha} g(b)\right. \\
-\left[J_{\sqrt{a b}-}^{\alpha}(f g)(a)+J_{\sqrt{a b}+}^{\alpha}(f g)(b)\right.
\end{array}\right] \mid\right] \leq \frac{\|g\|_{\infty}\left(\ln \frac{b}{a}\right)^{\alpha+1}}{\Gamma(\alpha+1)} C_{1}(\alpha) \sup \left\{\left|f^{\prime}(a)\right|,\left|f^{\prime}(b)\right|\right\}
$$

where

$$
C_{1}(\alpha)=\int_{0}^{\frac{1}{2}} u^{\alpha}\left[a^{1-u} b^{u}+a^{u} b^{1-u}\right] d u
$$

with $\alpha>0$.

Proof. From Lemma 1 we have

$$
\begin{aligned}
& \left|f(\sqrt{a b})\left[J_{\sqrt{a b}-}^{\alpha} g(a)+J_{\sqrt{a b}+}^{\alpha} g(b)\right]-\left[J_{\sqrt{a b}-}^{\alpha}(f g)(a)+J_{\sqrt{a b}+}^{\alpha}(f g)(b)\right]\right| \\
\leq & \frac{1}{\Gamma(\alpha)}\left[\begin{array}{c}
\int_{a}^{\sqrt{a b}}\left(\int_{a}^{t}\left(\ln \frac{s}{a}\right)^{\alpha-1}|g(s)| \frac{d s}{s}\right)\left|f^{\prime}(t)\right| d t \\
+\int_{\sqrt{a b}}^{b}\left(\int_{t}^{b}\left(\ln \frac{b}{s}\right)^{\alpha-1}|g(s)| \frac{d s}{s}\right)\left|f^{\prime}(t)\right| d t
\end{array}\right] \\
\leq & \frac{\|g\|_{\infty}}{\Gamma(\alpha)}\left[\begin{array}{c}
\int_{a}^{\sqrt{a b}}\left(\int_{a}^{t}\left(\ln \frac{s}{a}\right)^{\alpha-1} \frac{d s}{s}\right)\left|f^{\prime}(t)\right| d t \\
+\int_{\sqrt{a b}}^{b}\left(\int_{t}^{b}\left(\ln \frac{b}{s}\right)^{\alpha-1} \frac{d s}{s}\right)\left|f^{\prime}(t)\right| d t
\end{array}\right] .
\end{aligned}
$$

Setting $t=a^{1-u} b^{u}$ and $d t=a^{1-u} b^{u} \ln \left(\frac{b}{a}\right) d u$ gives

$$
\begin{aligned}
& \left|f(\sqrt{a b})\left[J_{\sqrt{a b}-}^{\alpha} g(a)+J_{\sqrt{a b}+}^{\alpha} g(b)\right]-\left[J_{\sqrt{a b}-}^{\alpha}(f g)(a)+J_{\sqrt{a b}+}^{\alpha}(f g)(b)\right]\right| \\
\leq & \frac{\|g\|_{\infty}}{\Gamma(\alpha)}\left[\begin{array}{c}
\int_{0}^{\frac{1}{2}}\left(\int_{a}^{a^{1-u} b^{u}}\left(\ln \frac{s}{a}\right)^{\alpha-1} \frac{d s}{s}\right)\left|f^{\prime}\left(a^{1-u} b^{u}\right)\right|\left(a^{1-u} b^{u}\right) \ln \left(\frac{b}{a}\right) d u \\
+\int_{\frac{1}{2}}^{1}\left(\int_{a^{1-u} b^{u}}^{b}\left(\ln \frac{b}{s}\right)^{\alpha-1} \frac{d s}{s}\right)\left|f^{\prime}\left(a^{1-u} b^{u}\right)\right|\left(a^{1-u} b^{u}\right) \ln \left(\frac{b}{a}\right) d u
\end{array}\right] \\
= & \frac{\|g\|_{\infty}}{\Gamma(\alpha)}\left[\begin{array}{c}
\int_{0}^{\frac{1}{2}}\left(\left.\frac{\left(\ln \frac{s}{a}\right)^{\alpha}}{\alpha}\right|_{a} ^{a^{1-u} b^{u}}\right)\left|f^{\prime}\left(a^{1-u} b^{u}\right)\right|\left(a^{1-u} b^{u}\right) \ln \left(\frac{b}{a}\right) d u \\
+\int_{\frac{1}{2}}^{1}\left(\left.\frac{-\left(\ln \frac{b}{s}\right)^{\alpha}}{\alpha}\right|_{a^{1-u} b^{u}} ^{b}\right)\left|f^{\prime}\left(a^{1-u} b^{u}\right)\right|\left(a^{1-u} b^{u}\right) \ln \left(\frac{b}{a}\right) d u
\end{array}\right] \\
= & \frac{\|g\|_{\infty}\left(\ln \frac{b}{a}\right)^{\alpha+1}}{\Gamma(\alpha+1)}\left[\begin{array}{c}
\int_{0}^{\frac{1}{2}} u^{\alpha}\left|f^{\prime}\left(a^{1-u} b^{u}\right)\right|\left(a^{1-u} b^{u}\right) d u \\
+\int_{\frac{1}{2}}^{1}(1-u)^{\alpha}\left|f^{\prime}\left(a^{1-u} b^{u}\right)\right|\left(a^{1-u} b^{u}\right) d u
\end{array}\right] .
\end{aligned}
$$

Since $\left|f^{\prime}\right|$ is quasi-geometrically convex on $[a, b]$, we have

$$
\left|f^{\prime}\left(a^{1-u} b^{u}\right)\right| \leq \sup \left\{\left|f^{\prime}(a)\right|,\left|f^{\prime}(b)\right|\right\} .
$$

If we use (2.2) in (2.1), we have

$$
\begin{aligned}
& \left|f(\sqrt{a b})\left[J_{\sqrt{a b}-}^{\alpha} g(a)+J_{\sqrt{a b}+}^{\alpha} g(b)\right]-\left[J_{\sqrt{a b}-}^{\alpha}(f g)(a)+J_{\sqrt{a b}+}^{\alpha}(f g)(b)\right]\right| \\
\leq & \frac{\|g\|_{\infty}\left(\ln \frac{b}{a}\right)^{\alpha+1}}{\Gamma(\alpha+1)}\left[\begin{array}{c}
\int_{0}^{\frac{1}{2}} u^{\alpha} \sup \left\{\left|f^{\prime}(a)\right|,\left|f^{\prime}(b)\right|\right\}\left(a^{1-u} b^{u}\right) d u \\
+\int_{\frac{1}{2}}^{1}(1-u)^{\alpha} \sup \left\{\left|f^{\prime}(a)\right|,\left|f^{\prime}(b)\right|\right\}\left(a^{1-u} b^{u}\right) d u
\end{array}\right] \\
= & \frac{\|g\|_{\infty}\left(\ln \frac{b}{a}\right)^{\alpha+1}}{\Gamma(\alpha+1)}\left[\begin{array}{c}
\int_{0}^{\frac{1}{2}} u^{\alpha}\left(a^{1-u} b^{u}\right) d u \\
+\int_{0}^{\frac{1}{2}} u^{\alpha}\left(a^{u} b^{1-u}\right) d u
\end{array}\right] \sup \left\{\left|f^{\prime}(a)\right|,\left|f^{\prime}(b)\right|\right\} \\
= & \frac{\|g\|_{\infty}\left(\ln \frac{b}{a}\right)^{\alpha+1}}{\Gamma(\alpha+1)}\left[\int_{0}^{\frac{1}{2}} u^{\alpha}\left[a^{1-u} b^{u}+a^{u} b^{1-u}\right] d u\right] \sup \left\{\left|f^{\prime}(a)\right|,\left|f^{\prime}(b)\right|\right\} .
\end{aligned}
$$


This completes the proof.

Corollary 1. In Theorem 5:

(1) If we take $\alpha=1$ we have the following Hermite-Hadamard-Fejer inequality for quasi-geometrically convex functions which is related to the left-hand side of (1.3):

$$
\left|f(\sqrt{a b}) \int_{a}^{b} \frac{g(x)}{x} d x-\int_{a}^{b} \frac{f(x) g(x)}{x} d x\right| \leq\|g\|_{\infty} \ln ^{2}\left(\frac{b}{a}\right) C_{1}(1) \sup \left\{\left|f^{\prime}(a)\right|,\left|f^{\prime}(b)\right|\right\},
$$

(2) If we take $g(x)=1$ we have the following Hermite-Hadamard inequality for quasi-geometrically convex functions in fractional integral forms which is related to the left-hand side of (1.4):

$$
\left|f(\sqrt{a b})-\frac{\Gamma(\alpha+1)}{2^{1-\alpha}\left(\ln \frac{b}{a}\right)^{\alpha}}\left[J_{\sqrt{a b}-}^{\alpha} f(a)+J_{\sqrt{a b}+}^{\alpha} f(b)\right]\right| \leq \frac{\ln \left(\frac{b}{a}\right)}{2^{1-\alpha}} C_{1}(\alpha) \sup \left\{\left|f^{\prime}(a)\right|,\left|f^{\prime}(b)\right|\right\},
$$

(3) If we take $\alpha=1$ and $g(x)=1$ we have the following Hermite-Hadamard inequality for quasi-geometrically convex functions:

$$
\left|f(\sqrt{a b})-\frac{1}{\ln \frac{b}{a}} \int_{a}^{b} \frac{f(x)}{x} d x\right| \leq \ln \left(\frac{b}{a}\right) C_{1}(1) \sup \left\{\left|f^{\prime}(a)\right|,\left|f^{\prime}(b)\right|\right\} .
$$

Theorem 6. Let $f: I \subset(0, \infty) \rightarrow \mathbb{R}$ be a differentiable function on $I^{\circ}$, the interior of $I$, such that $f^{\prime} \in L[a, b]$, where $a, b \in I$ and $a<b$. If $\left|f^{\prime}\right|^{q}, q \geq 1$, is quasi-geometrically convex on $[a, b], g:[a, b] \rightarrow \mathbb{R}$ is continuous and geometrically symmetric with respect to $\sqrt{a b}$, then the following inequality for fractional integrals holds:

$$
\left.\left[\begin{array}{c}
f(\sqrt{a b})\left[J_{\sqrt{a b}-}^{\alpha} g(a)+J_{\sqrt{a b}+}^{\alpha} g(b)\right. \\
-\left[J_{\sqrt{a b}-}^{\alpha}(f g)(a)+J_{\sqrt{a b}+}^{\alpha}(f g)(b)\right.
\end{array}\right] \mid\right] \leq \frac{\|g\|_{\infty} \ln ^{\alpha+1}\left(\frac{b}{a}\right)(\alpha+1)^{\frac{1}{q}-1}}{2^{(\alpha+1)\left(1-\frac{1}{q}\right)} \Gamma(\alpha+1)} C_{2}(\alpha)\left[\sup \left\{\left|f^{\prime}(a)\right|^{q},\left|f^{\prime}(b)\right|^{q}\right\}\right]^{\frac{1}{q}}
$$

where

$$
C_{2}(\alpha)=\left[\int_{0}^{\frac{1}{2}} u^{\alpha}\left(a^{1-u} b^{u}\right)^{q} d u\right]^{\frac{1}{q}}+\left[\int_{\frac{1}{2}}^{1}(1-u)^{\alpha}\left(a^{1-u} b^{u}\right)^{q} d u\right]^{\frac{1}{q}}
$$

with $\alpha>0$.

Proof. Using Lemma 1, we have

$$
\begin{aligned}
& \left|f(\sqrt{a b})\left[J_{\sqrt{a b}-}^{\alpha} g(a)+J_{\sqrt{a b}+}^{\alpha} g(b)\right]-\left[J_{\sqrt{a b}-}^{\alpha}(f g)(a)+J_{\sqrt{a b}+}^{\alpha}(f g)(b)\right]\right| \\
\leq & \frac{1}{\Gamma(\alpha)}\left[\begin{array}{c}
\int_{a}^{\sqrt{a b}}\left(\int_{a}^{t}\left(\ln \frac{s}{a}\right)^{\alpha-1}|g(s)| \frac{d s}{s}\right)\left|f^{\prime}(t)\right| d t \\
+\int_{\sqrt{a b}}^{b}\left(\int_{t}^{b}\left(\ln \frac{b}{s}\right)^{\alpha-1}|g(s)| \frac{d s}{s}\right)\left|f^{\prime}(t)\right| d t
\end{array}\right] \\
\leq & \frac{\|g\|_{\infty}}{\Gamma(\alpha)}\left[\begin{array}{c}
\int_{a}^{\sqrt{a b}}\left(\int_{a}^{t}\left(\ln \frac{s}{a}\right)^{\alpha-1} \frac{d s}{s}\right)\left|f^{\prime}(t)\right| d t \\
+\int_{\sqrt{a b}}^{b}\left(\int_{t}^{b}\left(\ln \frac{b}{s}\right)^{\alpha-1} \frac{d s}{s}\right)\left|f^{\prime}(t)\right| d t
\end{array}\right] .
\end{aligned}
$$

Setting $t=a^{1-u} b^{u}$ and $d t=a^{1-u} b^{u} \ln \left(\frac{b}{a}\right) d u$ gives

$$
\begin{aligned}
& \left|f(\sqrt{a b})\left[J_{\sqrt{a b}-}^{\alpha} g(a)+J_{\sqrt{a b}+}^{\alpha} g(b)\right]-\left[J_{\sqrt{a b}-}^{\alpha}(f g)(a)+J_{\sqrt{a b}+}^{\alpha}(f g)(b)\right]\right| \\
& \leq \frac{\|g\|_{\infty}}{\Gamma(\alpha)}\left[\begin{array}{c}
\int_{0}^{\frac{1}{2}}\left(\int_{a}^{a^{1-u} b^{u}}\left(\ln \frac{s}{a}\right)^{\alpha-1} \frac{d s}{s}\right)\left|f^{\prime}\left(a^{1-u} b^{u}\right)\right|\left(a^{1-u} b^{u}\right) \ln \left(\frac{b}{a}\right) d u \\
+\int_{\frac{1}{2}}^{1}\left(\int_{a^{1-u} b^{u}}^{b}\left(\ln \frac{b}{s}\right)^{\alpha-1} \frac{d s}{s}\right)\left|f^{\prime}\left(a^{1-u} b^{u}\right)\right|\left(a^{1-u} b^{u}\right) \ln \left(\frac{b}{a}\right) d u
\end{array}\right] \\
& =\frac{\|g\|_{\infty} \ln \left(\frac{b}{a}\right)}{\Gamma(\alpha)}\left[\begin{array}{c}
\int_{0}^{\frac{1}{2}}\left(\int_{a}^{a^{1-u} b^{u}}\left(\ln \frac{s}{a}\right)^{\alpha-1} \frac{d s}{s}\right)\left|f^{\prime}\left(a^{1-u} b^{u}\right)\right|\left(a^{1-u} b^{u}\right) d u \\
+\int_{\frac{1}{2}}^{1}\left(\int_{a^{1-u} b^{u}}^{b}\left(\ln \frac{b}{s}\right)^{\alpha-1} \frac{d s}{s}\right)\left|f^{\prime}\left(a^{1-u} b^{u}\right)\right|\left(a^{1-u} b^{u}\right) d u
\end{array}\right] .
\end{aligned}
$$


Using power mean inequality we have

$$
\begin{aligned}
& \left|f(\sqrt{a b})\left[J_{\sqrt{a b}-}^{\alpha} g(a)+J_{\sqrt{a b}+}^{\alpha} g(b)\right]-\left[J_{\sqrt{a b}-}^{\alpha}(f g)(a)+J_{\sqrt{a b+}}^{\alpha}(f g)(b)\right]\right| \\
\leq & \frac{\|g\|_{\infty} \ln \left(\frac{b}{a}\right)}{\Gamma(\alpha)}\left[\begin{array}{c}
{\left[\int_{0}^{\frac{1}{2}}\left(\int_{a}^{a^{1-u} b^{u}}\left(\ln \frac{s}{a}\right)^{\alpha-1} \frac{d s}{s}\right) d u\right]^{1-\frac{1}{q}}} \\
\times\left[\int_{0}^{\frac{1}{2}}\left(\int_{a}^{a^{1-u} b^{u}}\left(\ln \frac{s}{a}\right)^{\alpha-1} \frac{d s}{s}\right)\left|f^{\prime}\left(a^{1-u} b^{u}\right)\right|^{q}\left(a^{1-u} b^{u}\right)^{q} d u\right]^{\frac{1}{q}} \\
+\left[\int_{\frac{1}{2}}^{1}\left(\int_{a^{1-u} b^{u}}^{b}\left(\ln \frac{b}{s}\right)^{\alpha-1} \frac{d s}{s}\right) d u\right]^{1-\frac{1}{q}} \\
\times\left[\int_{\frac{1}{2}}^{1}\left(\int_{a^{1-u} b^{u}}^{b}\left(\ln \frac{b}{s}\right)^{\alpha-1} \frac{d s}{s}\right)\left|f^{\prime}\left(a^{1-u} b^{u}\right)\right|^{q}\left(a^{1-u} b^{u}\right)^{q} d u\right]^{\frac{1}{q}}
\end{array}\right] \\
& \frac{\left.\|g\|_{\infty} \ln \alpha+\frac{b}{a}\right)}{2^{(\alpha+1)\left(1-\frac{1}{q}\right)}(\alpha+1)^{1-\frac{1}{q}} \Gamma(\alpha+1)} \\
& \times\left[\begin{array}{c}
{\left[\int_{0}^{\frac{1}{2}} u^{\alpha}\left|f^{\prime}\left(a^{1-u} b^{u}\right)\right|^{q}\left(a^{1-u} b^{u}\right)^{q} d u\right]^{\frac{1}{q}}} \\
\left.+\left[\int_{\frac{1}{2}}^{1}(1-u)^{\alpha}\left|f^{\prime}\left(a^{1-u} b^{u}\right)\right|^{q}\left(a^{1-u} b^{u}\right)^{q} d u\right]^{\frac{1}{q}}\right] .
\end{array}\right.
\end{aligned}
$$

Since $\left|f^{\prime}\right|^{q}$ is quasi-geometrically convex on $[a, b]$, we know that for $u \in[0,1]$

$$
\left|f^{\prime}\left(a^{1-u} b^{u}\right)\right|^{q} \leq \sup \left\{\left|f^{\prime}(a)\right|^{q},\left|f^{\prime}(b)\right|^{q}\right\} .
$$

If we use (2.4) in (2.3), we have

$$
\begin{aligned}
& \left|f(\sqrt{a b})\left[J_{\sqrt{a b}-}^{\alpha} g(a)+J_{\sqrt{a b}+}^{\alpha} g(b)\right]-\left[J_{\sqrt{a b}-}^{\alpha}(f g)(a)+J_{\sqrt{a b}+}^{\alpha}(f g)(b)\right]\right| \\
\leq & \frac{\|g\|_{\infty} \ln ^{\alpha+1}\left(\frac{b}{a}\right)}{2^{(\alpha+1)\left(1-\frac{1}{q}\right)}(\alpha+1)^{1-\frac{1}{q}} \Gamma(\alpha+1)} \\
& \times\left[\begin{array}{c}
{\left[\int_{0}^{\frac{1}{2}} u^{\alpha} \sup \left\{\left|f^{\prime}(a)\right|^{q},\left|f^{\prime}(b)\right|^{q}\right\}\left(a^{1-u} b^{u}\right)^{q} d u\right]^{\frac{1}{q}}} \\
\left.+\left[\int_{\frac{1}{2}}^{1}(1-u)^{\alpha} \sup \left\{\left|f^{\prime}(a)\right|^{q},\left|f^{\prime}(b)\right|^{q}\right\}\left(a^{1-u} b^{u}\right)^{q} d u\right]^{\frac{1}{q}}\right]
\end{array}\right. \\
= & \frac{\|g\|_{\infty} \ln ^{\alpha+1}\left(\frac{b}{a}\right)}{2^{(\alpha+1)\left(1-\frac{1}{q}\right)}(\alpha+1)^{1-\frac{1}{q}} \Gamma(\alpha+1)}\left[\sup \left\{\left|f^{\prime}(a)\right|^{q},\left|f^{\prime}(b)\right|^{q}\right\}\right]^{\frac{1}{q}} \\
= & \frac{\left.\|\left[\int_{0}^{\frac{1}{2}} u^{\alpha}\left(a^{1-u} b^{u}\right)^{q} d u\right]^{\frac{1}{q}}+\left[\int_{\frac{1}{2}}^{1}(1-u)^{\alpha}\left(a^{1-u} b^{u}\right)^{q} d u\right]^{\frac{1}{q}}\right]}{2^{(\alpha+1)\left(1-\frac{1}{q}\right)}(\alpha+1)^{1-\frac{1}{q}} \Gamma(\alpha+1)}\left[\sup \left\{\left|f^{\prime}(a)\right|^{q},\left|f^{\prime}(b)\right|^{q}\right\}\right]^{\frac{1}{q}} \\
& \times\left[\left[\int_{0}^{\frac{1}{2}} u^{\alpha}\left(a^{1-u} b^{u}\right)^{q} d u\right]^{\frac{1}{q}}+\left[\int_{\frac{1}{2}}^{1}(1-u)^{\alpha}\left(a^{1-u} b^{u}\right)^{q} d u\right]^{\frac{1}{q}}\right]
\end{aligned}
$$

This completes the proof.

\section{Corollary 2. In Theorem 6:}

(1) If we take $\alpha=1$ we have the following Hermite-Hadamard-Fejer inequality for quasi-geometrically convex functions which is related to the left-hand side of (1.3):

$$
\left|f(\sqrt{a b}) \int_{a}^{b} \frac{g(x)}{x} d x-\int_{a}^{b} \frac{f(x) g(x)}{x} d x\right| \leq \frac{\|g\|_{\infty} \ln ^{2}\left(\frac{b}{a}\right)}{2^{3\left(1-\frac{1}{q}\right)}} C_{2}(1)\left[\sup \left\{\left|f^{\prime}(a)\right|^{q},\left|f^{\prime}(b)\right|^{q}\right\}\right]^{\frac{1}{q}},
$$

(2) If we take $g(x)=1$ we have the following Hermite-Hadamard inequality for quasi-geometrically convex functions in fractional integral forms which is related to the left-hand side of (1.4):

$$
\left|f(\sqrt{a b})-\frac{\Gamma(\alpha+1)}{2^{1-\alpha}\left(\ln \frac{b}{a}\right)^{\alpha}}\left[J_{\sqrt{a b}-}^{\alpha} f(a)+J_{\sqrt{a b}+}^{\alpha} f(b)\right]\right| \leq \frac{\ln \left(\frac{b}{a}\right)}{2^{2-\frac{\alpha+1}{q}}(\alpha+1)^{1-\frac{1}{q}}} C_{2}(\alpha)\left[\sup \left\{\left|f^{\prime}(a)\right|^{q},\left|f^{\prime}(b)\right|^{q}\right\}\right]^{\frac{1}{q}},
$$


(3) If we take $\alpha=1$ and $g(x)=1$ we have the following Hermite-Hadamard type inequality for quasi-geometrically convex functions:

$$
\left|f(\sqrt{a b})-\frac{1}{\ln \frac{b}{a}} \int_{a}^{b} \frac{f(x)}{x} d x\right| \leq \frac{\ln \left(\frac{b}{a}\right)}{2^{3\left(1-\frac{1}{q}\right)}} C_{2}(1)\left[\sup \left\{\left|f^{\prime}(a)\right|^{q},\left|f^{\prime}(b)\right|^{q}\right\}\right]^{\frac{1}{q}} .
$$

Theorem 7. Let $f: I \subset(0, \infty) \rightarrow \mathbb{R}$ be a differentiable function on $I^{\circ}$, the interior of $I$, such that $f^{\prime} \in L[a, b]$, where $a, b \in I$ and $a<b$. If $\left|f^{\prime}\right|^{q}, q>1$, is quasi-geometrically convex on $[a, b], g:[a, b] \rightarrow \mathbb{R}$ is continuous and geometrically symmetric with respect to $\sqrt{a b}$, then the following inequalities for fractional integrals holds:

$$
\begin{aligned}
& \left|f(\sqrt{a b})\left[J_{\sqrt{a b}-}^{\alpha} g(a)+J_{\sqrt{a b+}}^{\alpha} g(b)\right]-\left[J_{\sqrt{a b}-}^{\alpha}(f g)(a)+J_{\sqrt{a b}+}^{\alpha}(f g)(b)\right]\right| \leq \frac{\|g\|_{\infty} a \ln ^{\alpha+1-\frac{1}{q}}\left(\frac{b}{a}\right)}{2^{\frac{\alpha p+1}{p}}(\alpha p+1)^{\frac{1}{p}} q^{\frac{1}{q}} \Gamma(\alpha+1)} \\
& \times\left[\sup \left\{\left|f^{\prime}(a)\right|^{q},\left|f^{\prime}(b)\right|^{q}\right\}\right]^{\frac{1}{q}}\left[\left[\left(\frac{b}{a}\right)^{\frac{q}{2}}-1\right]^{\frac{1}{q}}+\left[\left(\frac{b}{a}\right)^{q}-\left(\frac{b}{a}\right)^{\frac{q}{2}}\right]\right],
\end{aligned}
$$

with $\alpha>0$ and $1 / p+1 / q=1$.

Proof. Using Lemma 1, Hölder's inequality and (2.4), setting $t=a^{1-u} b^{u}$ and $d t=a^{1-u} b^{u} \ln \left(\frac{b}{a}\right) d u$, we have

$$
\begin{aligned}
& \left|f(\sqrt{a b})\left[J_{\sqrt{a b}-}^{\alpha} g(a)+J_{\sqrt{a b}+}^{\alpha} g(b)\right]-\left[J_{\sqrt{a b}-}^{\alpha}(f g)(a)+J_{\sqrt{a b}+}^{\alpha}(f g)(b)\right]\right| \\
& \leq \frac{1}{\Gamma(\alpha)}\left[\begin{array}{c}
\int_{a}^{\sqrt{a b}}\left(\int_{a}^{t}\left(\ln \frac{s}{a}\right)^{\alpha-1}|g(s)| \frac{d s}{s}\right)\left|f^{\prime}(t)\right| d t \\
+\int_{\sqrt{a b}}^{b}\left(\int_{t}^{b}\left(\ln \frac{b}{s}\right)^{\alpha-1}|g(s)| \frac{d s}{s}\right)\left|f^{\prime}(t)\right| d t
\end{array}\right] \\
& \leq \frac{\|g\|_{\infty}}{\Gamma(\alpha)}\left[\begin{array}{c}
\int_{a}^{\sqrt{a b}}\left(\int_{a}^{t}\left(\ln \frac{s}{a}\right)^{\alpha-1} \frac{d s}{s}\right)\left|f^{\prime}(t)\right| d t \\
+\int_{\sqrt{a b}}^{b}\left(\int_{t}^{b}\left(\ln \frac{b}{s}\right)^{\alpha-1} \frac{d s}{s}\right)\left|f^{\prime}(t)\right| d t
\end{array}\right] \\
& =\frac{\|g\|_{\infty}}{\Gamma(\alpha)}\left[\begin{array}{c}
\int_{0}^{\frac{1}{2}}\left(\int_{a}^{a^{1-u} b^{u}}\left(\ln \frac{s}{a}\right)^{\alpha-1} \frac{d s}{s}\right)\left|f^{\prime}\left(a^{1-u} b^{u}\right)\right|\left(a^{1-u} b^{u}\right) \ln \left(\frac{b}{a}\right) d u \\
+\int_{\frac{1}{2}}^{1}\left(\int_{a^{1-u} b^{u}}^{b}\left(\ln \frac{b}{s}\right)^{\alpha-1} \frac{d s}{s}\right)\left|f^{\prime}\left(a^{1-u} b^{u}\right)\right|\left(a^{1-u} b^{u}\right) \ln \left(\frac{b}{a}\right) d u
\end{array}\right] \\
& =\frac{\|g\|_{\infty} \ln \left(\frac{b}{a}\right)}{\Gamma(\alpha)}\left[\begin{array}{c}
\int_{0}^{\frac{1}{2}}\left(\int_{a}^{a^{1-u} b^{u}}\left(\ln \frac{s}{a}\right)^{\alpha-1} \frac{d s}{s}\right)\left|f^{\prime}\left(a^{1-u} b^{u}\right)\right|\left(a^{1-u} b^{u}\right) d u \\
+\int_{\frac{1}{2}}^{1}\left(\int_{a^{1-u} b^{u}}^{b}\left(\ln \frac{b}{s}\right)^{\alpha-1} \frac{d s}{s}\right)\left|f^{\prime}\left(a^{1-u} b^{u}\right)\right|\left(a^{1-u} b^{u}\right) d u
\end{array}\right] \\
& \leq \frac{\|g\|_{\infty} \ln \left(\frac{b}{a}\right)}{\Gamma(\alpha)}\left[\begin{array}{c}
\left(\int_{0}^{\frac{1}{2}}\left(\int_{a}^{a^{1-u} b^{u}}\left(\ln \frac{s}{a}\right)^{\alpha-1} \frac{d s}{s}\right)^{p} d u\right)^{\frac{1}{p}} \\
\times\left(\int_{0}^{\frac{1}{2}}\left|f^{\prime}\left(a^{1-u} b^{u}\right)\right|^{q}\left(a^{1-u} b^{u}\right)^{q} d u\right)^{\frac{1}{q}} \\
+\left(\int_{\frac{1}{2}}^{1}\left(\int_{a^{1-u} b^{u}}^{b}\left(\ln \frac{b}{s}\right)^{\alpha-1} \frac{d s}{s}\right)^{p} d u\right)^{\frac{1}{p}} \\
\times\left(\int_{\frac{1}{2}}^{1}\left|f^{\prime}\left(a^{1-u} b^{u}\right)\right|^{q}\left(a^{1-u} b^{u}\right)^{q} d u\right)^{\frac{1}{q}}
\end{array}\right] \\
& =\frac{\|g\|_{\infty} \ln ^{\alpha+1}\left(\frac{b}{a}\right)}{2^{\frac{\alpha p+1}{p}}(\alpha p+1)^{\frac{1}{p}} \Gamma(\alpha+1)}\left[\begin{array}{c}
\left(\int_{0}^{\frac{1}{2}}\left|f^{\prime}\left(a^{1-u} b^{u}\right)\right|^{q}\left(a^{1-u} b^{u}\right)^{q} d u\right)^{\frac{1}{q}} \\
+\left(\int_{\frac{1}{2}}^{1}\left|f^{\prime}\left(a^{1-u} b^{u}\right)\right|^{q}\left(a^{1-u} b^{u}\right)^{q} d u\right)^{\frac{1}{q}}
\end{array}\right] \\
& \leq \frac{\|g\|_{\infty} \ln ^{\alpha+1}\left(\frac{b}{a}\right)}{2^{\frac{\alpha p+1}{p}}(\alpha p+1)^{\frac{1}{p}} \Gamma(\alpha+1)}\left[\begin{array}{c}
\left(\int_{0}^{\frac{1}{2}} \sup \left\{\left|f^{\prime}(a)\right|^{q},\left|f^{\prime}(b)\right|^{q}\right\}\left(a^{1-u} b^{u}\right)^{q} d u\right)^{\frac{1}{q}} \\
+\left(\int_{\frac{1}{2}}^{1} \sup \left\{\left|f^{\prime}(a)\right|^{q},\left|f^{\prime}(b)\right|^{q}\right\}\left(a^{1-u} b^{u}\right)^{q} d u\right)^{\frac{1}{q}}
\end{array}\right] \\
& =\frac{\|g\|_{\infty} a \ln ^{\alpha+1-\frac{1}{q}}\left(\frac{b}{a}\right)}{2^{\frac{\alpha p+1}{p}}(\alpha p+1)^{\frac{1}{p}} q^{\frac{1}{q}} \Gamma(\alpha+1)}\left[\sup \left\{\left|f^{\prime}(a)\right|^{q},\left|f^{\prime}(b)\right|^{q}\right\}\right]^{\frac{1}{q}} \\
& \times\left[\left[\left(\frac{b}{a}\right)^{\frac{q}{2}}-1\right]^{\frac{1}{q}}+\left[\left(\frac{b}{a}\right)^{q}-\left(\frac{b}{a}\right)^{\frac{q}{2}}\right]^{\frac{1}{q}}\right] .
\end{aligned}
$$


This completes the proof.

Corollary 3. In Theorem 7;

(1) If we take $\alpha=1$ we have the following Hermite-Hadamard-Fejer inequality for quasi-geometrically convex functions which is related to the left-hand side of (1.3):

$$
\begin{aligned}
& \left|f(\sqrt{a b}) \int_{a}^{b} \frac{g(x)}{x} d x-\int_{a}^{b} \frac{f(x) g(x)}{x} d x\right| \leq \frac{\|g\|_{\infty} a \ln ^{2-\frac{1}{q}}\left(\frac{b}{a}\right)}{2^{\frac{p+1}{p}}(p+1)^{\frac{1}{p}} q^{\frac{1}{q}}}\left[\sup \left\{\left|f^{\prime}(a)\right|^{q},\left|f^{\prime}(b)\right|^{q}\right\}\right]^{\frac{1}{q}} \\
& \times\left[\left[\left(\frac{b}{a}\right)^{\frac{q}{2}}-1\right]^{\frac{1}{q}}+\left[\left(\frac{b}{a}\right)^{q}-\left(\frac{b}{a}\right)^{\frac{q}{2}}\right]^{\frac{1}{q}}\right],
\end{aligned}
$$

(2) If we take $g(x)=1$ we have the following Hermite-Hadamard inequality for quasi-geometrically convex functions in fractional integral forms which is related to the left-hand side of (1.4):

$$
\begin{aligned}
& \left|f(\sqrt{a b})-\frac{\Gamma(\alpha+1)}{2^{1-\alpha}\left(\ln \frac{b}{a}\right)^{\alpha}}\left[J_{\sqrt{a b}-}^{\alpha} f(a)+J_{\sqrt{a b}+}^{\alpha} f(b)\right]\right| \leq \frac{a \ln ^{1-\frac{1}{q}}\left(\frac{b}{a}\right)}{2^{\frac{\alpha p+1}{p}+1-\alpha}(\alpha p+1)^{\frac{1}{p}} q^{\frac{1}{q}}}\left[\sup \left\{\left|f^{\prime}(a)\right|^{q},\left|f^{\prime}(b)\right|^{q}\right\}\right]^{\frac{1}{q}} \\
& \times\left[\left[\left(\frac{b}{a}\right)^{\frac{q}{2}}-1\right]^{\frac{1}{q}}+\left[\left(\frac{b}{a}\right)^{q}-\left(\frac{b}{a}\right)^{\frac{q}{2}}\right]^{\frac{1}{q}}\right],
\end{aligned}
$$

(3) If we take $\alpha=1$ and $g(x)=1$ we have the following Hermite-Hadamard inequality for quasi-geometrically convex functions:

$$
\begin{aligned}
& \left|f(\sqrt{a b})-\frac{1}{\ln \frac{b}{a}} \int_{a}^{b} \frac{f(x)}{x} d x\right| \leq \frac{a \ln ^{1-\frac{1}{q}}\left(\frac{b}{a}\right)}{2^{\frac{p+1}{p}}(p+1)^{\frac{1}{p}} q^{\frac{1}{q}}}\left[\sup \left\{\left|f^{\prime}(a)\right|^{q},\left|f^{\prime}(b)\right|^{q}\right\}\right]^{\frac{1}{q}} \\
& \times\left[\left[\left(\frac{b}{a}\right)^{\frac{q}{2}}-1\right]^{\frac{1}{q}}+\left[\left(\frac{b}{a}\right)^{q}-\left(\frac{b}{a}\right)^{\frac{q}{2}}\right]^{\frac{1}{q}}\right] .
\end{aligned}
$$

\section{References}

[1] L. Fejér, Uber die Fourierreihen, II. Math. Naturwise. Anz Ungar. Akad., Wiss 24 (1906), 369-390, (in Hungarian).

[2] J. Hadamard, Étude sur les propriétés des fonctions entières et en particulier d'une fonction considérée par Riemann. J. Math. Pures Appl. 58 (1893), 171-215.

[3] İ. İşcan, Hermite-Hadamard-Fejer type inequalities for convex functions via fractional integrals, arXiv preprint arXiv:1404.7722 (2014).

[4] İ. İşcan, Generalization of different type integral inequalities for s-convex functions via fractional integrals. Applicable Analysis(2013), doi: 10.1080/00036811.2013.851785.

[5] İ. İşcan, New general integral inequalities for quasi-geometrically convex functions via fractional integrals. J. Inequal. Appl. (2013), 2013(491).

[6] İ. İşcan, On generalization of different type integral inequalities for $s$-convex functions via fractional integrals. Mathematical Sciences and Applications E-Notes 2(1) (2014), 55-67.

[7] A. A. Kilbas, H. M. Srivastava, J. J. Trujillo, Theory and applications of fractional differential equations. Elsevier. Amsterdam, 2006.

[8] M. Kunt, İ. İşcan, On new inequalities of Hermite-Hadamard-Fejer type for GA-convex functions via fractional integrals. RGMIA Research Report Collection 18(2015), Article 108, 12 pp.

[9] M. A. Latif, S. S. Dragomir and E. Momaniat, Some Fejer type integral inequalities for geometricallyarithmetically-convex functions with applications. RGMIA Research Report Collection 18(2015), Article 25, $18 \mathrm{pp}$. 
[10] C. P. Niculescu, Convexity according to the geometric mean. Math. Inequal. Appl. 3 (2) (2000), 155-167. Available online at http:/ /dx.doi.org/10.7153/mia-03-19.

[11] C. P. Niculescu, Convexity according to means. Math. Inequal. Appl. 6 (4) (2003), 571-579. Available online at http://dx.doi.org/10.7153/mia-06-53.

[12] M.Z. Sarıkaya, On new Hermite Hadamard Fejér type integral inequalities. Stud. Univ. Babeş-Bolyai Math. 57(3) (2012), 377-386.

[13] Erhan Set, İ. İşcan, M. Zeki Sarikaya, M. Emin Ozdemir, On new inequalities of Hermite-Hadamard-Fejer type for convex functions via fractional integrals. Applied Mathematics and Computation 259 (2015) 875-881.

[14] K.-L. Tseng, G.-S. Yang and K.-C. Hsu, Some inequalities for differentiable mappings and applications to Fejér inequality and weighted trapezoidal formula. Taiwanese journal of Mathematics 15(4) (2011), 1737-1747.

[15] J. Wang, X. Li, M. Fečkan and Y. Zhou, Hermite-Hadamard-type inequalities for Riemann-Liouville fractional integrals via two kinds of convexity. Appl. Anal. 92(11) (2012), 2241-2253. doi:10.1080/00036811.2012.727986.

[16] J. Wang, C. Zhu and Y. Zhou, New generalized Hermite-Hadamard type inequalities and applications to special means. J. Inequal. Appl. (2013), 2013(325), 15 pages.

\section{Affiliations}

MeHMET KunT

ADDRESS: Karadeniz Technical University, Dept. of Mathematics, 61080, Trabzon-Turkey.

E-MAIL: mkunt@ktu.edu.tr

İMDAT İşCAN

AdDRESS: Giresun University, Dept. of Mathematics, 28200, Giresun-Turkey.

E-MAIL: imdati@yahoo.com 